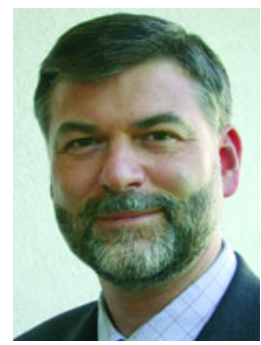

Thomas Jahn
Störungen motorischer Funktionen bei schizophren erkrankten Patienten Katatone Symptome und Soft Signs

Thomas Jahn

Klinik und Poliklinik für Psychiatrie und Psychotherapie der Technischen Universität München

psychoneuro 2005; 31 (7+8): 365-373

$\mathrm{D}$ ie bei schizophren erkrankten Patienten auftretenden Störungen motorischer Funktionen umfassen drei Phänomenkreise:

- medikamentenbedingte Bewegungsstörungen, die vor allem unter konventionellen Neuroleptika auftreten (s. Beitrag von Dose in diesem Heft)

- katatone Symptome, die schon vor der neuroleptischen Ära beschrieben wurden und eine Unterform der Schizophrenie charakterisieren, und

- so genannte Neurological Soft Signs, eine nur unscharf definierte Klasse motorischer $\mathrm{Ab}$ normitäten, die im Rahmen von Studien erst in den letzten Jahren breitere Aufmerksamkeit erfahren hat.

Diese drei Gruppen von Symptomen sind nicht eindeutig voneinander abzugrenzen (Abb. 1).

Beispielsweise wird der Tremor häufig als vorübergehende Begleiterscheinung einer Neuroleptikabehandlung angesehen, obwohl das gleiche Phänomen in kaum einer präneuroleptischen Beschreibung der Katatonie fehlt und neuerdings auch bei der Einschätzung von Soft Signs zu berücksichtigen ist.

Motorische Störungen bei schizophren erkrankten Patienten können in drei teilweise überlappende Gruppen von Symptomen unterteilt werden: neuroleptika-induzierte Bewegungsstörungen, katatone Symptome und diskrete motorische Anomalien im Sinne neurologischer Soft Signs. Dieser Artikel beschreibt Definition und Umfang katatoner Symptome und motorischer Soft Signs bei schizophrenen Erkrankungen und referiert neuere Forschungsergebnisse, insbesondere zu Prävalenzraten in verschiedenen diagnostischen Populationen, klinischen Korrelaten und möglichen Ursachen. Zusätzlich werden verbesserte Untersuchungsmethoden beschrieben, wie standardisierte Rating-Skalen und computergestützte Methoden der kinematischen Bewegungsanalyse. Während katatone Symptome für die klinische Praxis relevant bleiben, stellen motorische Soft Signs einen zunehmend faszinierenden Gegenstand für die ätiologische Grundlagenforschung dar.

Im Folgenden wird der aktuelle Wissensstand zu den nicht pharmakogenen motorischen Symptomen der Schizophrenie - also katatone Symptome und motorische Soft Signs - referiert. Dabei wird auch auf neuere Methoden zur deren Untersuchung eingegangen.

\section{Katatone Symptome}

Der Begriff der Katatonie ist eng mit dem der Schizophrenie verknüpft, seitdem Kraepelin die von Kahlbaum 1874 als „Spannungsirresein" beschriebene Krankheitsentität zu einem der Subtypen der Dementia praecox erklärt hatte (11). Katatone Schizophrenien scheinen allerdings im Laufe der Jahrzehnte immer seltener geworden zu sein. Lag ihr Anteil an der stationären Aufnahmeprävalenz in nahezu allen Untersuchungen vor 1960 über 25\%, so ist dieser Anteil seither auf 2-8\% gesunken. Als Gründe hierfür werden häufig genannt:

- Ausschluss von Hirnerkrankungen durch verbesserte differentialdiagnostische Möglichkeiten

- Rückgang der im ersten Viertel des 20. Jahrhunderts häufigen Encephalitis lethargica

- Fortschritt in der rehabilitationsorientierten Behandlung auch chronisch kranker Patienten.

Auch wenn diese Faktoren eine Rolle spielen mögen, scheint der Rückgang der Katatonie aber doch weniger ein epidemiologisches Faktum als ein diagnostisches Artefakt zu sein.

Katatone Symptome sind bei schizophren erkrankten Patienten häufig

In einer Untersuchung an 91 chronisch schizophren erkrankten 


\section{Abb. 1 Symptomgruppen motorischer Störungen bei schizophrenen Erkrankungen}

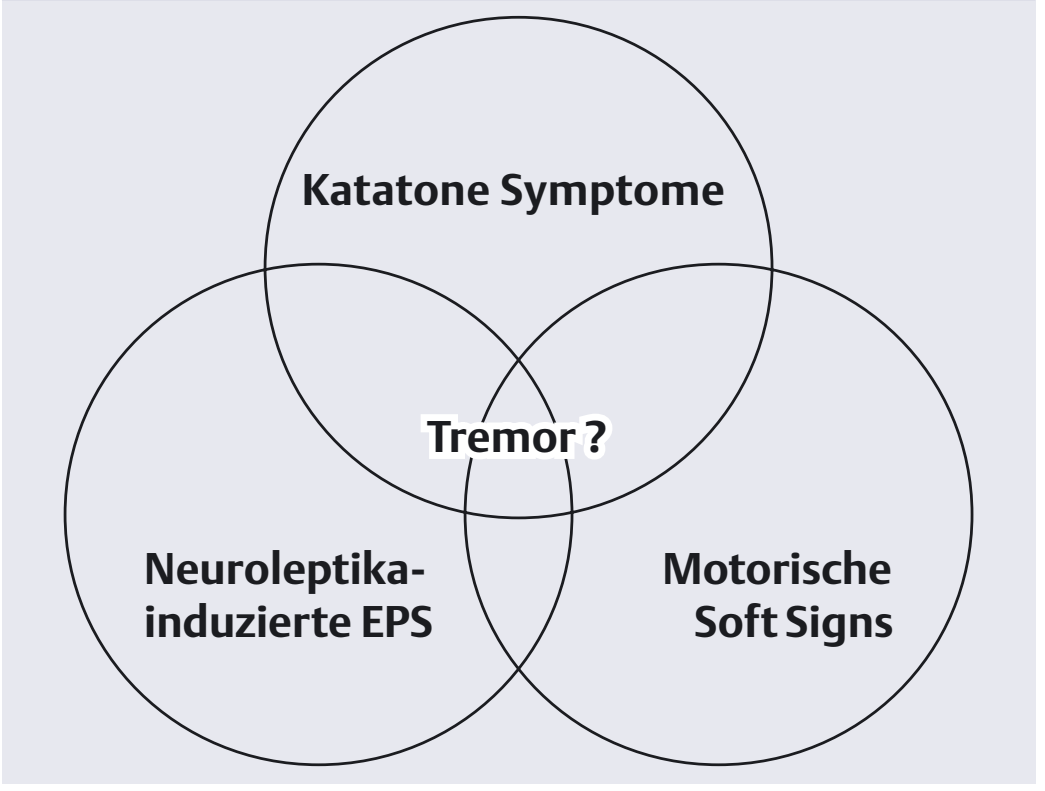

Patienten (8) erhielten nach ICD-10 Kriterien 9,9\%, nach präneuroleptischem Katatonie-Verständnis 33\% der Klientel die Diagnose einer katatonen Schizophrenie. Wurde dieses Stichprobendrittel erneut nach ICD10 diagnostiziert, so mussten $70 \%$ der traditionellen Katatonien einem anderen als dem katatonen Subtyp zugeordnet werden. Die Gründe hierfür ergaben sich aus der Analyse der Häufigkeitsverteilung der einzelnen katatonen Symptome. Von den nach traditionellen Gesichtspunkten insgesamt 41 identifizierbaren motorischen Anomalien waren in dieser Studie am häufigsten Bewegungsstereotypien (63,3\%), Parakinesen $(60,0 \%)$, fehlende Ausdrucksbewegungen $(60,0 \%)$, Haltungsanomalien (56,7\%), Gegengreifen $(43,3 \%)$ und Stupor/Mutismus $(43,3 \%)$. Davon werden im ICD-10 nur Stupor/Mutismus berücksichtigt.

Diese Ergebnisse wie auch die anderer Autoren demonstrieren, dass auch unter heutigen Behandlungsbedingungen ein bedeutsamer Prozentsatz schizophren erkrankter Patienten eine Häufung katatoner Symptome aufweist, ohne dass dies zu einer entsprechenden Diagnose führt, da die in den gegenwärtigen Klassifikationssystemen genannten katatonen
Symptome nur einen Ausschnitt der tatsächlich vorhandenen Störungen darstellen (Abb. 2).

\section{Katatone Symptome sind reliabel erfassbar}

Diese und andere Beobachtungen der letzten Jahre haben die Beschäftigung mit katatonen Symptomen reintensiviert, wie zahlreiche neuere Publikationen belegen $(2,4$, 5, 16, 21, 24, 25). Im Zuge dessen wurden auch spezielle Untersuchungsinstrumente zur standardisierten Erfassung katatoner Symptome entwickelt (Tab. 1).

Diese Skalen knüpfen an die differenzierte Katatoniediagnostik der klassischen Psychiatrie an. So können beispielsweise mit der KRS auch subtile Veränderungen katatoner Symptome erfasst werden. Insgesamt besitzen alle in Tabelle 1 aufgeführten Skalen befriedigende bis gute psychometrische Eigenschaften. Unterschiede bestehen u.a. hinsichtlich der Itemzusammenstellung, der Kriterien für die quantitative Einschätzung der Symptomschwere sowie der Schwellenwerte zur Diagnose eines katatonen Syndroms. Eine allgemein akzeptierte Definition katatoner Symptome und verbindliche Standards zu ihrer Erfassung müssen erst noch erarbeitet werden.

\section{Katatone Symptome sind} nosologisch unspezifisch

Trotz der historisch engen Bindung des Katatoniebegriffes an die Schizophrenie kommen katatone Zustände besonders häufig im Rahmen affektiver Erkrankungen vor. Etwa 25\% aller manisch erkrankten Patienten erfüllen zumindest vorübergehend die DSM-Kriterien einer Katatonie, und mehr als die Hälfte der Patienten, die als kataton einzustufen sind, leiden an einer manisch-depressiven Erkrankung. Insbesondere bei Manien sind katatone Symptome umso wahrscheinlicher, je schwerer die Psychose ist. Insofern stellen katatone Symptome einen für den Verlauf und die Prognose manischer Episoden relevanten Schweregradindikator dar $(11,18)$.

Katatone Symptome sind jedoch nicht auf schizophrene und affektive Erkrankungen beschränkt, da die möglichen organischen Ursachen außerordentlich vielfältig sind (16). Dazu zählen Infektionskrankheiten (neben der bereits genannten Encephalitis lethargica z.B. auch Herpes simplex), vaskuläre Störungen (Basilarthrombosen), metabolische Prozesse (Hyperparathyroidismus, hepatitische Enzephalopathie, Kohlenmonoxid-Vergiftung), Drogen (neben Neuroleptika unter anderem Alkohol, Lithium, Amphetamine, Steroide), Hirngewebsläsionen (des Hirnstammes und der Ganglien, Wernicke Enzephalopathie, Sklerose) und Epilepsien (nonkonvulsiver epileptischer Status). Die Vielzahl dieser und anderer Konditionen muss differentialdiagnostisch berücksichtigt werden.

Über Ursachen katatoner Symptome, die nicht auf bekannte organische Erkrankungen zurückgeführt werden können, ist derzeit noch wenig bekannt. Bisher vorliegende Befunde umfassen (eher geringgradige) Atrophien des Hirnstammes und des Cerebellums, Hypoperfusionen des linken inferioren Temporallappens unter Einschluss von Teilen des limbischen Systems, Asymmetrien im Glukose-Metabolismus der Basalganglien, sowie Dysfunktionen des mit den Basalganglien eng verbundenen ventralen striatal-pallidalen Komplexes als Teil eines mesotelenzephalen 
Dopamin-Systems. Die Komplexität vieler katatoner Symptome, die auch auf Störungen der Aufmerksamkeit und der Handlungsintention hinweisen, lassen weiträumige Verursachungsmodelle unter Einschluss frontaler und subkortikaler Areale (Basalganglien, Dienzephalon, Hirnstamm) - vermutlich verbunden mit einem mesolimbisch-mesostriären dopaminergen Ungleichgewicht - adäquater erscheinen als allzu eng fokussierende Lokalisationsversuche. Völlig offen ist die Frage der Verursachung katatoner Symptome in solchen Fällen, die mit keiner hirnorganischen oder psychiatrischen Störung in Zusammenhang stehen (idiopathische Katatonie).

\section{Katatone Symptome bilden möglicherweise ein eigenstän- diges diagnostisches Syndrom}

In einer aktuellen Übersicht reflektieren Taylor und Fink (25) die Stellung der Katatonie im Rahmen der gegenwärtigen psychiatrischen Klassifikationssysteme vor dem Hintergrund der folgenden Studienergebnisse.

- Die Katatonie ist ein ätiologisch heterogenes, gleichwohl klar definiertes Syndrom aus bestimmten motorischen Symptomen und Verhaltensanomalien, das reliabel diagnostiziert werden kann.

- Obwohl mehr als 40 motorische Symptome der Katatonie bekannt sind, genügt die Präsenz von mindestens zwei prominenten Symptomen über 24 Stunden, um das Syndrom zuverlässig zu identifizieren.

- Grob zu unterscheiden sind retardiert-stuporöse Formen von erregt-deliranten.

- Ungefähr $10 \%$ aller akut erkrankten Patienten, die sich in stationäre psychiatrische Behandlung begeben, weisen Zeichen einer Katatonie auf; dabei ist die Prävalenz bei affektiv erkrankten (insbesondere manischen) $\mathrm{Pa}$ tienten höher als bei schizophren erkrankten.

- Die Katatonie reagiert positiv auf spezifische Behandlungsansätze wie sedative Antikonvulsiva (Barbiturate, Benzodiazepine) und Elektrokonvulsionstherapie (EKT). Andererseits führen sowohl konventionelle wie atypische Neuroleptika meist zu einer deutlichen Zustandsverschlechterung bis hin zur malignen Form der Katatonie.

Aufgrund dieser Sachlage empfehlen die Autoren, die Katatonie etwa in Analogie zum Delir - als eigenständige Kategorie in künftige psychiatrische Diagnosesysteme aufzunehmen (Tab. 2).

\section{Motorische Soft Signs}

Innerhalb eines weiten Katatoniebegriffes wurden bereits um 1900 auch ausgesprochen diskrete motorische Störungen beschrieben $(9,11)$. In neuerer Zeit belegen Untersuchungen zu neurologischen Soft Signs bei schizophren erkrankten Patienten (6), dass neben sensorischen und integrativen Funktionen vor allem motorische Funktionen beeinträchtigt sind, von eher basalen motorischen Koordinationsleistungen (z.B. Diadochokinese, Finger-Nase-Versuch)

\section{Abb. 2 Symptome der Katatonie}

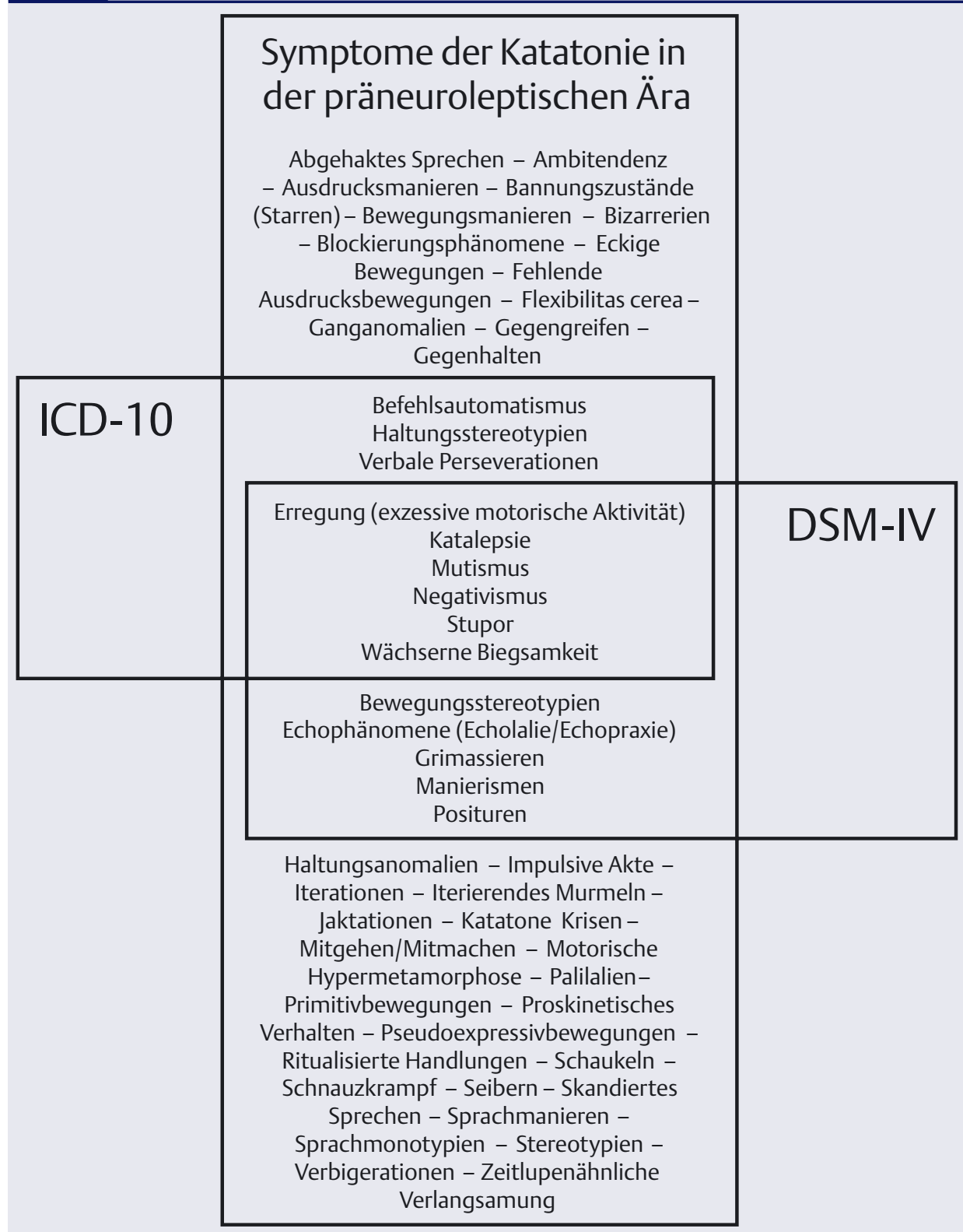

Symptome der Katatonie bzw. des katatonen Subtyps der Schizophrenie in der präneuroleptischen Ära und nach den derzeit geltenden operationalen Diagnosesystemen 
bis zu komplexen Bewegungssequenzen (z.B. Finger-Daumen-Opposition, Faust-Kante-Ballen-Probe). Diskrete motorische Störungen als Teilmenge neurologischer Soft Signs können wie diese insgesamt - als Beeinträchtigungen definiert werden, die

- nicht schon im Alltagsverhalten der Patienten grob auffällig sind

- keine eindeutigen Rückschlüsse auf lokalisierbare Schädigungen des peripheren oder zentralen Nervensystems erlauben und

- dennoch wahrscheinlich eine neurologische Ursache haben.

Soft Signs sind bei schizophren erkrankten Patienten häufig und nicht als neuroleptisches Epiphänomen erklärbar

Die sich aus zahlreichen Untersuchungen (9) ergebenden mittleren Prävalenzraten neurologischer Soft Signs liegen bei schizophren erkrankten Patienten mit 60 bis 70\% rund zehnmal höher als bei Gesunden (um 6\%) und deutlich höher als bei anderen psychiatrischen Patienten (30 bis 40\%). Bei dimensionaler Betrachtung (Anzahl positiver Zeichen bzw. Summenwert aus mehrstufigen Bewertungen) weisen schizophren erkrankte Patienten ohne Ausnahme signifikant mehr bzw. ausgeprägtere Soft Signs als Gesunde auf, meist auch gegenüber anderen
Patientengruppen. Soziodemografische Variablen (Alter, Geschlecht, Bildungsniveau, Schichtzugehörigkeit) sind mit Soft Signs nicht korreliert. Negative Korrelationen mit dem Intelligenzniveau wurden wiederholt berichtet, erklären aber höchstens 15\% der Varianz.

Nur vereinzelt fanden sich $\mathrm{Zu}$ sammenhänge mit dem bisherigen Krankheitsverlauf ( prämorbide Anpassung, Ersterkrankungsalter, Krankheitsdauer, Anzahl und Dauer früherer Hospitalisierungen). Schizoaffektive Patienten unterscheiden sich nicht von schizophren erkrankten. Auch bestehen kaum Unterschiede zwischen hospitalisierten

Tab. 1 Standardisierte Ratingskalen zur Erfassung katatoner Symptome (Quellenangaben in 11)

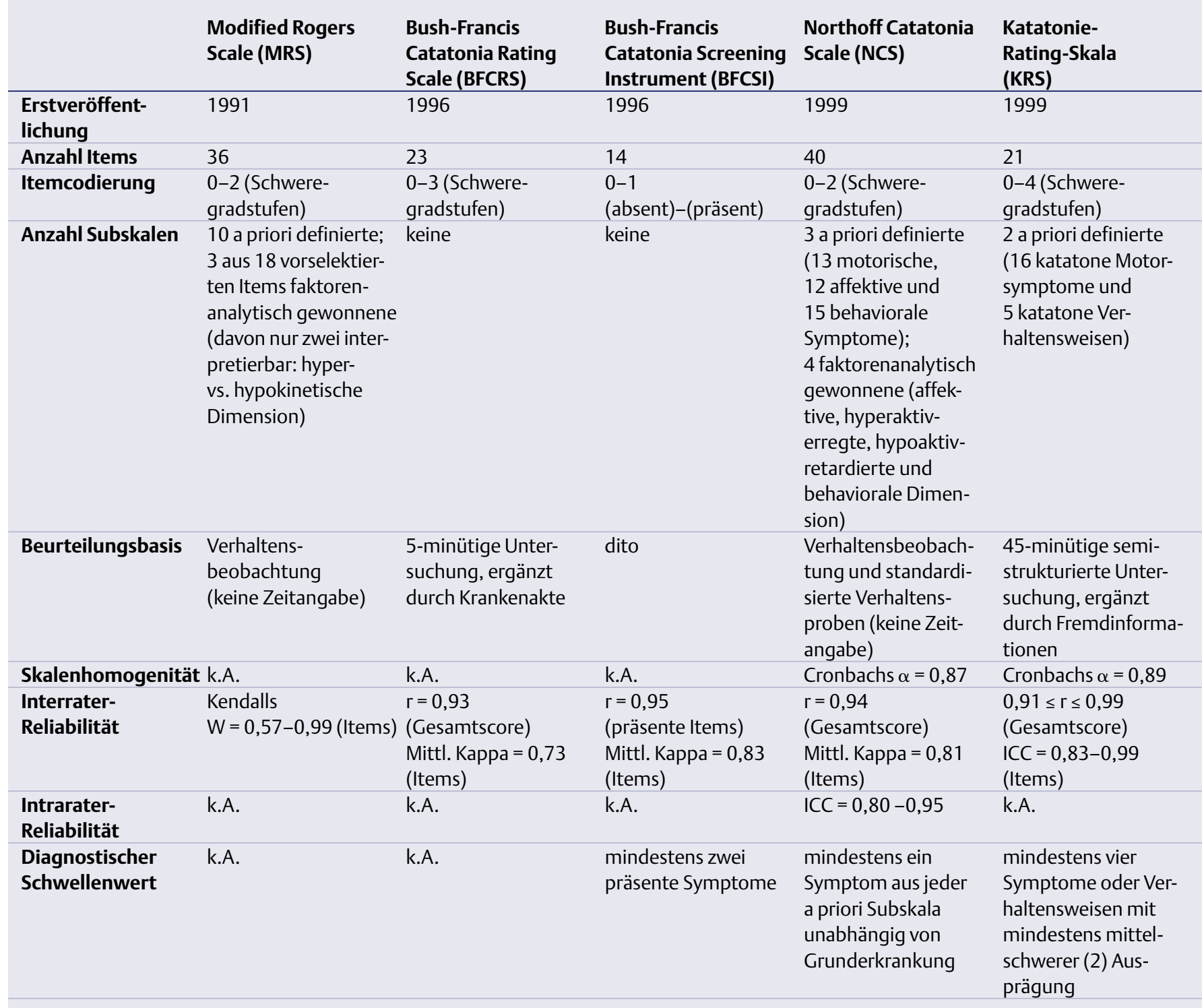


und nicht hospitalisierten Patienten, obwohl im Behandlungsverlauf eine Reduktion neurologischer Zeichen beschrieben wurde, wenn auch nicht auf das Niveau Gesunder (23). Soft Signs sind möglicherweise prädiktiv für einen ungünstigeren Krankheitsverlauf und dementsprechend ausgeprägter bei chronisch schizophren erkrankten Patienten.

Hervorzuheben ist, dass mit wenigen Ausnahmen bisher keine bedeutsamen Zusammenhänge zwischen Soft Signs und neuroleptischer Dosishöhe, neuroleptischer Expositionszeit und extrapyramidalmotorischen Medikamenten-Nebenwirkungen (EPS, TD) gefunden wurden. Auch die Art der neuroleptischen Medikation (konventionell vs. atypisch) scheint ohne Einfluss zu sein. Neurologische Soft Signs konnten auch bei Neuroleptika-naiven schizophren erkrankten Patienten vermehrt nachgewiesen werden.

Zusammenfassend sind Soft Signs bei schizophren erkrankten Patienten häufig, mit den meisten Krankheitsaspekten unkorreliert und nicht als Epiphänomen neuroleptischer Behandlung zu erklären $(1,6,9)$.

\section{Soft Signs beinhalten vor allem motorische Zeichen}

Auch zur standardisierten Erfassung von Soft Signs wurden geeignete Untersuchungsskalen entwickelt (Tab. 3). Im Unterschied zu ähnlichen Skalen, mit denen in der Kinder- und Jugendpsychiatrie Untersuchungen zum Konzept der „Minimalen zerebralen Dysfunktion" durchgeführt wurden, zielen diese Instrumente speziell auf die Erfassung von Soft Signs bei erwachsenen psychiatrischen Patienten.

Eigene systematische Itemanalysen haben gezeigt, dass gerade motorische Aufgaben zur klinischen Beurteilung neurologischer Soft Signs gute bis sehr gute psychometrische Eigenschaften aufweisen. Auf dieser Grundlage wurde mit der Brief Motor Scale (BMS) eine Ratingskala entwickelt und kreuzvalidiert, welche nur die für die Unterscheidung schizophren erkrankter Patienten von Gesunden diskriminationsstärksten motorischen Aufgaben zusammenfasst (13; deutsche Fassung: 10, Anhang $G$ ). Entsprechend gering ist die Stichprobenüberschneidung individueller BMS-Summenwerte von schizophren erkrankten Patienten und Gesunden (Abb. 3).

\section{Soft Signs korrelieren mit neuropsychologischen Defiziten und Hirnanomalien}

Obwohl mehrere Untersuchungen auf einen spezifischen Zusammenhang zwischen Soft Signs und negativen Symptomen der Schizophrenie hinweisen, sind die Befunde zu psychopathologischen Korrelaten insgesamt uneinheitlich. Speziell motorische Soft Signs korrelieren relativ hoch mit formalen, nicht aber mit inhaltlichen Denkstörungen.

Signifikante Zusammenhänge bestehen darüber hinaus mit einem ganzen Spektrum neuropsychologischer Leistungsbeeinträchtigungen, einschließlich sprachlicher und mnestischer Funktionen. Am häufigsten repliziert wurde ein Zusammenhang zwischen motorischen Soft Signs und exekutiven Leistungsdefiziten (z.B. Anzahl der perseverativen Fehler im Wisconsin Card Sorting Test).

Bildgebende Verfahren zeigen strukturell (CT, MRT) keine Zusammenhänge mit der Ventricle Brain Ratio (VBR), jedoch relativ konsis- tent mit Erweiterungen speziell des dritten Ventrikels und der kortikalen Sulci.

Funktionell (motorische Stimulation unter SPECT, PET, fMRT) fanden sich eine diffuse bilaterale $\mathrm{Hy}$ peraktivierung oder eine fehlende Aktivierung spezifischer, motorisch relevanter Hirnareale sowie Hinweise auf eine gestörte interhemisphärische bzw. frontokortikal-subkortikale Balance (7).

\section{Soft Signs kennzeichnen nicht} nur akut psychotische Patienten Retrospektive und prospektive (Längsschnitt-)Studien zeigen, dass diskrete motorische Störungen in vielen Fällen bereits lange vor Ausbruch der Psychose bestehen $(20,22)$. Ein origineller Untersuchungsansatz ist die Auswertung privater Videoaufnahmen aus der Kindheit schizophren erkrankter Personen (26). Aufnahmen aus verschiedenen Entwicklungsphasen der späteren Patienten wurden mit solchen ihrer gesund gebliebenen Geschwister hinsichtlich Alter und Spielsituation so weit wie möglich parallelisiert. Beim Betrachten der anonymisierten Videosequenzen waren überraschenderweise nicht nur Experten, sondern auch Laien in der Lage, überzufällig häufig diejenigen Kinder zu identifizieren, die später an einer Schizophrenie erkranken sollten. Die mehr-

\section{Tab. 2 Diagnostische Kriterien und Unterformen der Katatonie}

Diagnostische Leitsymptome

A) Immobilität, Mutismus oder Stupor von mindestens einer Stunde Dauer, zusammen mit mindestens einem der folgenden Symptome: Katalepsie, Befehlsautomatismus oder Haltungsanomalien, jeweils mindestens zweimalig beobachtet oder provoziert.

B) Bei Abwesenheit von Immobilität, Mutismus oder Stupor mindestens zwei der folgenden Symptome, jeweils mindestens zweimalig beobachtet oder provoziert: Stereotypien, Echophänomene, Katalepsie, Befehlsautomatismus, Haltungsanomalien, Negativismus, Gegenhalten, Ambitendenz.

\section{Unterformen}

Nicht-maligne Katatonie

(Kahlbaum-Syndrom)

Delirante Katatonie (delirante Manie, erregte Katatonie)

Maligne Katatonie (malignes neuroleptisches

Syndrom, Serotonin-Syndrom)
Optionale Zusatzspezifikationen

sekundär zu: einer affektiven Störung einer somatischen Grunderkrankung oder einem toxischem Status einer neurologischen Störung einer psychotischen Störung

Diagnostische Kriterien und Unterformen der Katatonie, wie sie in künftigen Revisionen psychiatrischer Klassifikationssysteme als eigenständige diagnostische Kategorie kodiert werden könnte $(5,25)$ 
fach replizierten Ergebnisse dieses auch als „archival-observational method" bezeichneten Forschungsansatzes konvergieren mit Befunden prospektiver Langzeitstudien an Kindern schizophren erkrankter Eltern, wonach etwa die Hälfte dieser Kinder motorische Soft Signs aufweisen, die durch verschiedene Entwicklungsphasen hindurch bis in die Adoleszenz und das Erwachsenenalter hinein persistieren und - zusammen mit unangepasstem Sozialverhalten und basalen Aufmerksamkeitsstörungen - eine gewisse prognostische Validität zur Vorhersage schizophrener Spektrumsstörungen haben. Passend hierzu belegen mehrere Studien erhöhte Prävalenzen motorischer Soft Signs bei erstgradig Verwandten von schizophren erkrankten Patienten sowie bei Cluster-A-Persönlichkeitsstörungen, insbesondere Schizotypie (9).
Mikrobehaviorale Analyse motorischer Soft Signs

Experimentell am besten untersucht sind basale okulomotorische Störungen, insbesondere Störungen langsamer Augenfolgebewegungen und fehlerhafte Antisakkaden (17). Obwohl zunächst nicht im Kontext neurologischer Soft Signs erörtert, können diese Störungen darunter subsummiert werden, sind sie doch

- ähnlich diskret, daher

- nur durch bestimmte Verhaltensaufgaben provozierbar

- den Betroffenen nicht bewusst

- nicht als Neuroleptikanebenwirkungen erklärbar und

- nicht einfach Folge einer mangelnden Aufmerksamkeitszuwendung oder anderer kognitiver Defizite.

Obwohl okulomotorische Störungen nicht spezifisch für die Schi- zophrenie sind, gibt es doch überzeugende Belege dafür, dass sie über einen genetischen Mechanismus mit der Schizophrenie in Verbindung stehen. Dabei gilt die Antisakkadenstörung aufgrund ihrer erheblich höheren Sensitivität derzeit als der geeignetere Marker (17).

Neben okulomotorischen werden auch manumotorische Koordinationsdefizite zunehmend experimentell untersucht. So belegen apparative Untersuchungen zum maximalschnellen Fingertapping nicht nur eine signifikante Verlangsamung schizophren erkrankter Patienten, sondern auch eine erhöhte intraindividuelle Variabilität der Tappingfrequenz (15). Bei auditiv zu synchronisierenden Tappingbewegungen ist die Synchronisationsgenauigkeit verringert und die Interresponse-Intervalle sind sehr variabel, was ältere Hypothesen $\mathrm{zu}$ einem

Tab. 3 Standardisierte Ratingskalen zur Erfassung neurologischer Soft Signs (Quellenangaben in 9, 10)

\begin{tabular}{|c|c|c|c|c|c|}
\hline & $\begin{array}{l}\text { Neurological } \\
\text { Evaluation Scale } \\
\text { (NES) }\end{array}$ & $\begin{array}{l}\text { Heidelberger } \\
\text { NSS-Skala } \\
\text { (HD-NSS) } \\
\end{array}$ & $\begin{array}{l}\text { Cambridge } \\
\text { Neurological } \\
\text { Inventory (CNI) }\end{array}$ & $\begin{array}{l}\text { Standardized } \\
\text { Neurological } \\
\text { Examination (SNE) }\end{array}$ & $\begin{array}{l}\text { Brief Motor Scale } \\
\text { (BMS) }\end{array}$ \\
\hline $\begin{array}{l}\text { Erstveröffent- } \\
\text { lichung }\end{array}$ & 1989 & 1993 & 1995 & 2000 & 2004 \\
\hline Anzahl Items & 26 (davon 14 bilateral) & 16 (davon 10 bilateral) & 80 (davon 11 bilateral) & 23 (davon 9 bilateral) & 10 (davon 6 bilateral) \\
\hline Itemcodierung & $\begin{array}{l}3 \text { Schweregradstufen } \\
(0-2)\end{array}$ & $\begin{array}{l}\text { 4 Schweregradstufen } \\
(0-3)\end{array}$ & $\begin{array}{l}\text { 4 Schweregradstufen } \\
(0,0,5,1,2)\end{array}$ & $\begin{array}{l}4 \text { Schweregradstufen } \\
(0-3)\end{array}$ & $\begin{array}{l}3 \text { Schweregradstufen } \\
(0-2)\end{array}$ \\
\hline & $\begin{array}{l}\text { tion, Motorische Ko- } \\
\text { ordination, Sequen- } \\
\text { zierung komplexer } \\
\text { Bewegungsfolgen, } \\
\text { Andere }\end{array}$ & & $\begin{array}{l}\text { Koordination, Sen- } \\
\text { sorische Integration, } \\
\text { Primitive Reflexe, } \\
\text { Tardive Dyskinesien, } \\
\text { Katatone Zeichen, } \\
\text { Extrapyramidale } \\
\text { Zeichen, Suppres- } \\
\text { sionsstörung }\end{array}$ & $\begin{array}{l}\text { Motorische Koordi- } \\
\text {-nation, Motorisch } \\
\text {-integrative Funktion, } \\
\text { Sensorische Integra- } \\
\text { tion, Unwillkürliche } \\
\text { Bewegungen und } \\
\text { Posituren, } \\
\text { Lateralisierung }\end{array}$ & $\begin{array}{l}\text { Motorische Koordi- } \\
\text { nation, Motorische } \\
\text { Sequenzierung }\end{array}$ \\
\hline Beurteilungsbasis & $\begin{array}{l}\text { 35-minütige, } \\
\text { standardisierte } \\
\text { Untersuchung }\end{array}$ & $\begin{array}{l}\text { standardisierte } \\
\text { Untersuchung }\end{array}$ & $\begin{array}{l}20 \text { bis } 40 \text {-minütige, } \\
\text { standardisierte } \\
\text { Untersuchung }\end{array}$ & $\begin{array}{l}\text { Standardisierte } \\
\text { Untersuchung } \\
\text { (keine Zeitangabe) }\end{array}$ & $\begin{array}{l}15 \text { bis } 20 \text {-minütige, } \\
\text { standardisierte } \\
\text { Untersuchung }\end{array}$ \\
\hline Skalenhomogenität & k.A. & Cronbachs $\alpha=0,85$ & k.A. & Cronbachs $\alpha=0,85$ & Cronbachs $\alpha=0,83$ \\
\hline $\begin{array}{l}\text { Retest- } \\
\text { Reliabilität }\end{array}$ & k.A. & k.A. & k.A. & k.A. & $\begin{array}{l}r_{\mathrm{tt}}=0,84 \\
\text { (Gesamtscore) }\end{array}$ \\
\hline $\begin{array}{l}\text { Interrater- } \\
\text { Reliabilität }\end{array}$ & $\begin{array}{l}\mathrm{ICC}=0,95 \\
\text { (Gesamtscore) } \\
0,00 \leq \mathrm{ICC} \leq 1,00 \\
\text { (Items) }\end{array}$ & $\begin{array}{l}r=0,88 \\
\text { (Gesamtscore) }\end{array}$ & $\begin{array}{l}\text { Kendalls } \\
W=0,82-1,00 \\
\text { ( } 14 \text { ausgewählte } \\
\text { Items) }\end{array}$ & $\begin{array}{l}\text { ICC }=0,97 \\
\text { (Gesamtscore) } \\
0,40 \leq \text { Kappa } \leq 0,75 \\
\text { (meiste Items) }\end{array}$ & $\begin{array}{l}0,70 \leq \text { Kappa } \leq 1,00 \\
\text { (meiste Items) }\end{array}$ \\
\hline $\begin{array}{l}\text { Intrarater- } \\
\text { Reliabilität }\end{array}$ & k.A. & k.A. & k.A. & k.A. & k.A. \\
\hline
\end{tabular}


„redundanzassoziierten“ kognitiven Defizit schizophren erkrankter Patienten wiederbelebt. Interessanterweise scheint diese manumotorische Synchronisationsstörung mit formalen Denkstörungen einherzugehen. Detaillierte Analysen der Antizipationsintervalle beim synchronisierten Fingertapping deuten auf eine bei schizophren erkrankten Patienten im Vergleich zu Gesunden veränderte $40-\mathrm{Hz}$-Aktivität in einzelnen Strukturen des Gehirns. Die 40-Hz-Aktivität des Gehirns wird durch eine Reihe unterschiedlicher Neurotransmitter gesteuert, wobei sich insbesondere Veränderungen im dopaminergen und serotonergen System störend auswirken können.

Die genaueste Untersuchung motorischer Soft Signs ermöglichen computergestützte Methoden der kinematischen Bewegungsanalyse. Abbildung 4 illustriert das Fingertapping eines gesunden und zweier schizophren erkrankter Probanden (Instruktionsbedingung ,schnellstmöglich“), wobei die Beispiele a) und b) repräsentativ für die durchschnittlichen Leistungskennwerte (Frequenz, Amplitude, Variabilität der einzelnen Bewegungssegmente und ihrer kinematischen Parameter) sind, wie sie in einer eigenen Untersuchung an 26 stationär behandelten schizophren erkrankten Patienten und nach Alter, Geschlecht und Händigkeit parallelisierten gesunden Kontrollprobanden gefunden wurden. Die Darstellung unter c) ist demgegenüber ein extremes Beispiel für die Verlangsamung und die erhöhte Variabilität der Bewegungsausführung, die nach gruppenstatistischer Auswertung insgesamt kennzeichnend für schizophren erkrankte Patienten sind.

In drei eigenen kinematischen Tappingstudien mit zusammen 83 schizophren erkrankten Patienten und 117 nach Alter und Händigkeit gematchten gesunden Kontrollen wiesen gemessen am 10. bzw. 90. Perzentil der entsprechenden Kennwerteverteilung Gesunder 73,5\% der Patienten maximale Tappingleistungen auf, die durch abnorme Verlangsamung, abnorm erhöhte Variabilität oder beide Phänomene zugleich gekennzeichnet waren (15).

\section{Abb. 3 Stichprobenverteilungen individueller Summenwerte der Brief Motor Scale (BMS) bei schizophren erkrankten Patienten und gesunden Kontrollprobanden}

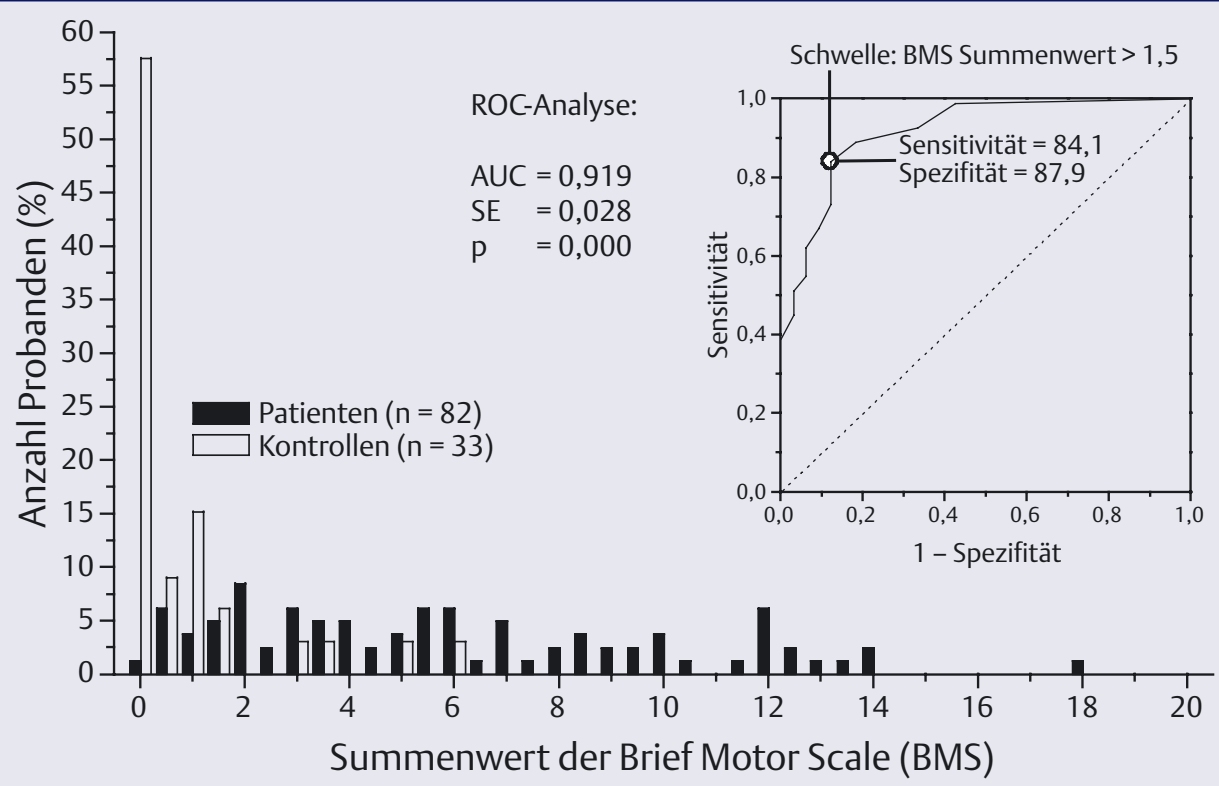

Die Receiver Operating Characteristic (ROC) zeigt für einen Schwellenwert von 1,5 eine rund $84 \%$ ige Sensitivität und $88 \%$ ige Spezifität dieses Instrumentes beim Nachweis motorischer Soft Signs schizophren Erkrankter (modifiziert nach 13).

Subgruppenunterschiede innerhalb des schizophren erkrankten Spektrums bestanden nicht, ebenso wenig signifikante Korrelationen mit klinischen Merkmalen wie Krankheitsdauer, Zahl der Hospitalisierungen, sozioökonomisches Funktionsniveau, Psychopathologie und abnormale unwillkürliche Bewegungen. Auch die neuroleptische Tagesdosis und klinisch eingeschätzte Medikamentennebenwirkungen korrelierten zwar signifikant, aber nur gering mit einzelnen kinematischen Parametern. Die höchste Korrelation ergab sich in einer Subgruppe von 43 Patienten für den $\mathrm{Zu}$ sammenhang zwischen Tappingrate und klinisch eingeschätzten motorischen Soft Signs auf einer Subskala der Neurological Evaluation Scale (NES; Sequencing of Complex Motor Acts: $r_{s}=-0,42, p=0,005$ ).

In einer anderen Serie eigener kinematischer Untersuchungen zur feinmotorischen Dysdiadochokinese beim repetitiven Zeichnen übereinandergelagerter Kreise auf einem Digitalisiertablett wurden ähnliche Ergebnisse gewonnen (9). Eine signifikant erhöhte intraindividuelle motorische Variabilität bei der feinmo- torischen Dysdiadochokinese zeigten auch psychiatrisch unbehandelte studentische Probanden mit ausgeprägten schizotypischen Persönlichkeitsmerkmalen im Vergleich zu nicht-schizotypen gleichaltrigen Kontrollen (14). Um künftig auch solche Aufgaben einer kinematischen Bewegungsanalyse zugänglich zu machen, die üblicherweise bei der klinischen Prüfung motorischer Soft Signs verwendet werden, wurde eine Untersuchungsbatterie entwickelt, die auf einer ultraschallgestützten Messtechnik beruht (10, Anhang H). Auf die damit gewonnenen Untersuchungsergebnisse kann an dieser Stelle nicht eingegangen werden (12).
Motorische Soft Signs sind ein möglicher phänotypischer Indi- kator für eine genetisch determi- nierte Krankheitsdisposition
Insgesamt ist festzuhalten, dass bei schizophren erkrankten Patien- ten mittels verschiedener Methoden ein ganzes Spektrum diskreter moto- rischer Koordinationsstörungen im Sinne motorischer Soft Signs nachge- wiesen werden kann. Vor allem Un- tersuchungen an Patientenangehöri- 


\section{Abb. 4 Kinematische Messanordnung und Beispiele für Fingertapping-Bewegungen}

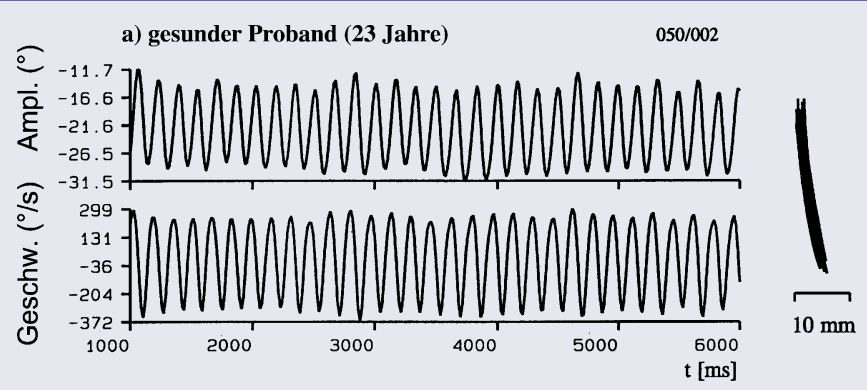

b) schizophren erkrankter Patient (22 Jahre)

$505 / 002$
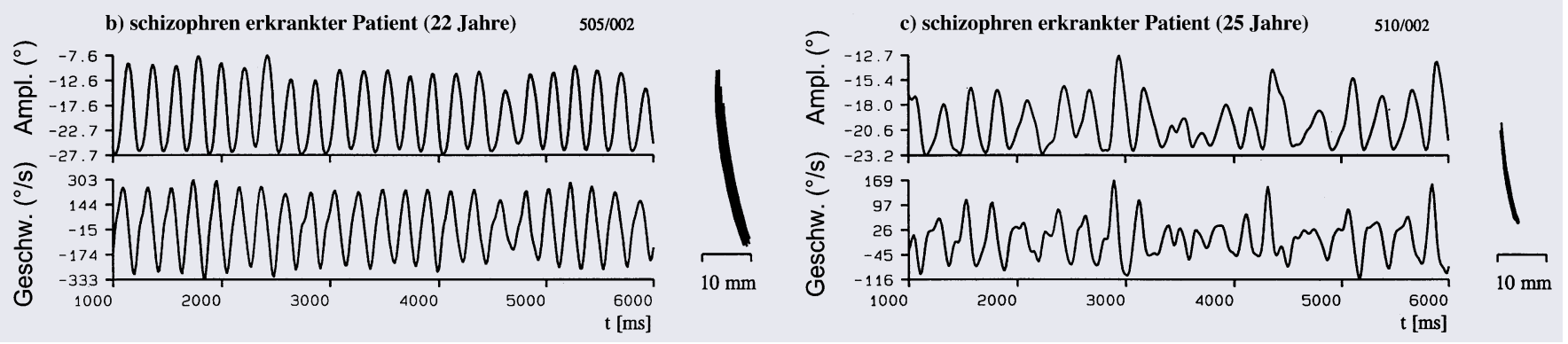

Dargestellt sind jeweils die Ortstrajektorie (horizontale Winkeländerung unter dem Zeigefinger) und darunter das Geschwindigkeitsprofil der Bewegung (Winkelgeschwindigkeit) über die Zeit sowie rechts die Auf- und Abwärtsbewegungen der Fingerspitzen in der sagittalen Ebene (modifiziert nach 15)

gen und an psychometrisch definierten schizotypen Personen sprechen dafür, dass motorische Soft Signs mehr sind als ein randständiger Befund der Schizophrenieliteratur. Entsprechend beschreibt die Schizophrenietheorie von Meehl (19) motorische Soft Signs wie die Dysdiadochokinese als substratnahe Indikatoren einer ubiquitären (bisher hypothetischen) minimalen Funktionsaberration bei der synaptischen Signalübertragung (synaptic slippage), aus der über einen mehrstufigen Prozess wechselseitiger Verschränkung genetischer und erfahrungsbedingter Faktoren eine spezifische Persönlichkeitsstruktur (schizotypy) mit subklinischen Denkstörungen (cognitive slippage), Anhedonie und sozialem Rückzug resultiert. Der Theorie zufolge sind solche Personen unter dem Einfluss äußerer und innerer Stressoren besonders vulnerabel für psychotische Dekompensationen. Die in prospektiven Längsschnittuntersuchungen an Kindern schizophrener Eltern gewonnenen Befunde zur prognostischen Validität motorischer Soft Signs bei der Vorhersage späterer schizophrener Spektrumsstörungen (einschließlich Cluster-A-Persönlichkeitsstörungen) sind mit dieser Sichtweise vereinbar.

\section{Schlussfolgerungen und Perpektiven}

Die motorischen Störungen schizophren erkrankter Patienten bilden drei Gruppen von Symptomen, die zunehmend besser differenziert, aber noch nicht vollständig verstanden werden können.

Unter dem Begriff der Katatonie wurden und werden höchst unterschiedliche Phänomene zusammengefasst, die von Beeinträchtigungen der motorischen Koordination bis zum lebensgefährlichen Stupor, von eng umgrenzten Haltungsanomalien bis zu komplexesten Handlungsritualen reichen. Es ist sehr unwahrscheinlich, dass diesen verschiedenartigen motorischen und psychomotorischen Anomalien eine einheitliche Ursache zugrunde liegt. Die seit Jahrzehnten konkurrierenden psychogenen vs. neurogenen Ansätze zur Interpretation und Erklärung katatoner Symptome verdeutlichen dies bis in die Gegenwart hinein, wenn auch die enge Beziehung vieler dieser Symptome zum extrapyramidalmotorischen System und zum Dopaminstoffwechsel unbestritten ist. Ähnlich wie „der“ Wahn, so scheint auch „die“ Katatonie ein zu allen bekannten nosologischen Entitäten quer liegendes Phänomenbündel zu sein, das eine Reihe traditioneller Dichotomien psychiatrischer Ordnungsversuche in Frage stellt. Gleichzeitig besteht wegen der oft schwierigen Abgrenzung pharmakogener von morbogenen Bewegungsstörungen das „katatone Dilemma“ (3) weiter fort - trotz heute vermehrtem Einsatz atypischer Neuroleptika.

Im Gegensatz dazu liegt die besondere Bedeutung der wesentlich unscheinbareren motorischen Koordinationsstörungen im Sinne neurologischer Soft Signs darin, dass sie kein Merkmal der Psychose als solcher sind, sondern einen relativ überdauernden, bereits prämorbid nachweisbaren Trait darstellen, der sehr wahrscheinlich eine diffuse neurobiologische Funktionsstörung indiziert, die mit der Vulnerabilität für schizophrene Spektrumsstörungen assoziiert zu sein scheint. In diesem Sinne haben sich die in den vergangenen Jahren unternommenen empirischen Untersuchungen zu neurologischen Soft Signs schizophren erkrankter Patienten von der Fokussierung der psychiatrischen Motorikforschung auf das lange Zeit dominierende Katatonieproblem gelöst und bilden heute einen eigenen Forschungsschwerpunkt $(1,9)$. 
Neben den neuroleptikabedingten Bewegungsstörungen bleiben also die katatonen Symptome - schon wegen der Gefahr ihrer Zuspitzung zu lebensbedrohlichen Zuständen - vor allem für den Kliniker relevant, während die motorischen Soft Signs einen für den ätiologisch orientierten Grundlagenforscher zunehmend faszinierenden Untersuchungsgegenstand darstellen. Motorische Soft Signs könnten künftig aber auch praktische Relevanz dadurch gewinnen, dass sie als zusätzliche Indikatoren in einen diagnostischen Prozess eingebracht werden, der mehr als bisher eine neurobiologisch valide Klassifikation psychopathologischer Syndrome anstrebt. Eine solche Klassifikation hätte sehr wahrscheinlich auch Konsequenzen für die Behandlung schizophrener Psychosen.

\section{Catatonic Symptoms and Soft Signs}

Motor disorders in schizophrenia can be subdivided into three partly overlapping categories: 1) neuroleptic-induced movement disorders, 2) catatonic symptoms, and 3) more minor motor anomalies as part of neurological soft signs. This article describes the definition and range of catatonic symptoms and motor soft signs in schizophrenia and summarizes some of the newer research findings, with special regard to prevalence rates in various diagnostic populations, clinical correlates and possible causations. Also, improvements in the assessment of catatonic symptoms and motor soft signs are described, such as the development of standardized rating scales and computerized methods for the kinematic analysis of motor performance. While catatonic symptoms remain an important topic for the clinician, motor soft signs have become a fascinating challenge for the neurobiologically oriented researcher.

\section{Key Words}

schizophrenia - motor disorders - catatonia - soft signs

\section{Literatur bei der Redaktion / im Internet unter www.psychoneuro.info}

\section{Korrespondenzadresse:}

Prof. Dr. phil. Dipl.-Psych. Thomas Jahn

Leiter Klinische und Experimentelle Neuropsychologie

Klinik und Poliklinik für Psychiatrie und Psychotherapie

Klinikum rechts der Isar

der Technischen Universität München

Ismaninger Straße 22

81675 München

th.jahn@Irz.tum.de

www.psykl.med.tum.de/neuropsy
Georg Thieme Verlag KG

Rüdigerstraße 14

70469 Stuttgart

Telefon (0711) 89 31-0

Telefax (0711) 89 31-322

Verantwortliche Schriftleitung:

Markus Gastpar, Essen

Jürgen Fritze, Pulheim

Erweiterte Schriftleitung:

Günther Faust, Mainz

Manfred Fichter, Prien am Chiemsee

Andreas Hufnagel, Essen

Joachim Morgner, Dresden

Götz-Erik Trott, Aschaffenburg

Wissenschaftlicher Beirat:

Thomas Becker, Günzburg; Stefan Bender, Marsberg; Horst Berzewski, Berlin; Lothar Blaha, Deggendorf; Hans-Dieter Brenner, Bern; Pasquale Calabrese, Bochum; Klemens Dieckhöfer, Bonn; Tilman Elliger, Köln; Volker Faust, Ravensburg; Franz joseph Freisleder, München; Michael Geyer, Leipzig; Ralf Gold, Göttingen; Iver Hand, Hamburg; Kurt Heinrich, Düsseldorf; Hamburg; Kunt ul Hoff, Zürich; Georg Juckel, Berlin; Siegfried Kaumeier, Merzig; Wilfried Kuhn, Schweinfurt; Gerd Laux, Wasserburg/München; Matthias R. Lemke, Bonn; Walter E. Müller, Frankfurt am Main; Thomas Müller, Bochum; Dieter Naber, Hamburg; Hartmut Radebold, Kas sel; Helmut Remschmidt, Marburg; Peter Riederer, Würzburg; Eckart Rüther, Göttingen; Jörn Peter Sieb, Stralsund; Wolfgang Weig, Osnabrück· Wolfgang Werner, Merzig: Roland Wörz Bad Schönborn; Manfred Wolfersdorf, Bayreuth

Bestellungen: Über den Buchhandel oder direkt beim Verlag

Bezugsbedingungen:

Einzelheft $€ 10,50+$ Porto.

Jahresabonnement $€ 75$,

inkl. MwSt. und Porto.

Für Medizinstudenten

gegen Nachweis und Bankeinzug

$€ 48,-$ inkl. MwSt. und Porto.

Ausland zuzüglich Versandkosten

(cash with order) $€ 19,80$ (Europa)

bzw. $€ 52,80$ (Airlift)

Der Abonnementpreis umfasst $12 \mathrm{Ka}$ lendermonate (Mindestlaufzeit). Abonnements laufen weiter, wenn nicht 3 Monate vor lahresende eine Abbestellung beim Verlag vorliegt.

Bei Nichtbelieferung im Falle höherer Gewalt, bei Störungen des Betriebsfriedens, Arbeitskampf (Streik, Aussperrung) bestehen keine Ansprüche gegen den Verlag.

Verlag und Copyright:

(c) 2005 by Georg Thieme Verlag,

Rüdigerstraße 14, 70469 Stuttgart.

Mit dem Abdruck des Beitrages erwirbt der Verlag das alleinige und ausschließ liche Recht für die Veröffentlichung in sämtlichen Publikumsmedien sowi Übersetzungen in fremde Sprachen. Nachdruck, fotomechanische Wiedergabe und Speicherung in den Datenverarbeitungsanlagen, auch auszugsweise, nu nach schriftlicher Genehmigung des Verlages. Die Abbildungen in den Beiträgen stammen, wenn nicht anders vermerkt, von den jeweiligen Autoren. Für Inhat, von den jeweiligen Autoren. Für Inhalt, Vorschau und Titelseite werden Abbildungen aus den beschriebenen Arbeite ebenfalls im Inhaltsverzeichnis deklariert. ebenfalls im Inhaltsver

Zur Veröffentlichung in der Zeitschrift anZurvomen werden unveröffentlich genommen werdentichte Originalarbeiten, Ubersichtsarbeiten und Kasuistiken, die nicht auch gleichzeitig an anderer Stelle zur Veröffentlichung eingereicht sein dürfen. Richtlinien zur Abfassung des Manuskriptes können bei de Redaktion angefordert werden.

Die eingereichten Arbeiten für Editorial, Schwerpunkt/Brennpunkt und Übersicht/Originalarbeit werden einem pee review der Schriftleitung unterzogen. Der Inhalt der übrigen Rubriken und de Supplements liegt in der Verantwortung der Redaktion.
Redaktionsleitung:

Günther Buck

Telefon (0711) 89 31-440

Chefredaktion:

Katrin Wolf

Telefon (0711) 89 31-179

Katrin.Wolf@thieme.de

Redaktionsassistenz:

Sabine Bischoff

Telefon (0711) 8931-5 51

Telefax (0711) 8931-322

Internet-Adresse:

http://www.psychoneuro.info

Verantwortlich für den Anzeigenteil:

pharmedia, Anzeigen-

und Verlagsservice $\mathrm{GmbH}$

Anzeigenleitung:

Greta Weller

Telefon (0711) 89 31-304

Greta.Weller@pharmedia.de

Zur Zeit gilt Anzeigenpreisliste

1.10 .2004

Zeitschriftenvertrieb:

Telefon (0711) 8931-321

Herstellung:

Werner Schulz

Telefon (0711) 8931-331

Satz, Grafik, Layout:

Wolfgang Eckl, Werner Schulz,

Karl-Heinz Zobel

Druck und Verarbeitung:

W. Kohlhammer Druckerei GmbH + Co. 70329 Stuttgart

Bankverbindung:

Deutsche Bank Stuttgart

Konto-Nr. 1420 017, BLZ 60070070

Landesbank Baden-Württemberg

Konto-Nr. 2055 723, BLZ 60050101

Postgiro Stuttgart

Konto-Nr. 45 000-705, BLZ 60010070

Erscheinungsweise: monatlich

Regularly listed in EMBASE

\section{Hinweis:}

Wie jede Wissenschaft ist die Medizin ständigen Entwicklungen unterworfen. Forschung und klinische Erfahrung erweitern unsere Erkenntnis, insbesondere was Behandlung, medikamentöse The rapie sowie Diagnostik (Laborwerte etc.) anbelangt. Soweit in dieser Zeitschrift Dosierungen, Applikationen oder Laborwerte erwähnt werden, darf der Lese zwar darauf vertrauen, dass Autoren Herausgeber und Verlag große Sorgfalt darauf verwandt haben, dass diese Angraun Wissensstand bei Fertigstegaben dem Wissensstand bei Fertigstellung entsprechen. Für Angaben übe Dosierungsanweisungen, Applikations 列 lag jedoch keine Gewähr übernommen werden. Jeder Benutzer ist angehalten, durch sorgfältige Prüfung der Beipackzettel der verwendeten Präparate und ggf. nach Konsultation eines Spezialisten festzustellen, ob die dort gegebene Empfehlung für Dosierungen oder die Beachtung von Kontraindikationen Beachtung von Kontraindikationen gegenuber der Angabe in dieser Zeitschrift abweicht. Eine solche Prüfung ist besonders wichtig bei selten verwendeten Präparaten oder solchen, die neu auf den Markt gebracht worden sind. Jede Dosierung oder Applikation erfolgt auf eigene Gefahr des Benutzers. Laborwerte müssen immer auf Ihre Plausibilität geprüft werden und sind abhängig vom jeweiligen Testgerät bzw. Testkit. Autoren und Verlag appellieren an jeden Benutzer, ihm auffallende Ungenauigkeiten dem Verlag mitzuteilen. 